\title{
REVIEWS \\ Do Statins Impair Cognition? A Systematic Review and Meta-Analysis of Randomized Controlled Trials
}

\author{
Brian R. Ott, MD' , Lori A. Daiello, PharmD, ScM' , Issa J. Dahabreh, MD, MS ${ }^{2,3}$, Beth A. Springate, PhD 4 , \\ Kimberly Bixby, $\mathrm{BS}^{7}$, Manjari Murali, $P \mathrm{PD}^{7}$, and Thomas A. Trikalinos, $\mathrm{MD}^{2,3}$ \\ 'Rhode Island Hospital, Department of Neurology, Alpert Medical School of Brown University, Providence, RI, USA; ${ }^{2}$ Center for Evidence-based \\ Medicine, School of Public Health, Brown University, Providence, RI, USA; ${ }^{3}$ Department of Health Services, Policy \& Practice, School of Public Health, \\ Brown University, Providence, RI, USA; ${ }^{4}$ Department of Psychiatry and Human Behavior, Rhode Island Hospital, Providence, RI, USA.
}

BACKGROUND: In 2012, the United States Food and Drug Administration (FDA) issued a warning regarding potential adverse effects of HMG-CoA reductase inhibitors (statins) on cognition, based on the Adverse Events Reporting System and a review of the medical literature. We aimed to synthesize randomized clinical trial (RCTs) evidence on the association between statin therapy and cognitive outcomes.

METHODS: We searched MEDLINE, EMBASE, and Cochrane CENTRAL through December 2012, and reviewed published systematic reviews of statin treatment. We sought RCTs that compared statin treatment versus placebo or standard care, and reported at least one cognitive outcome (frequency of adverse cognitive events or measurements using standard neuropsychological cognitive test scores). Studies reporting sufficient information to calculate effect sizes were included in metaanalyses. Standardized and unstandardized mean differences were calculated for continuous outcomes for global cognition and for pre-specified cognitive domains. The main outcome was change in cognition measured by neuropsychological tests; an outcome of secondary interest was the frequency of adverse cognitive events observed during follow-up.

RESULTS: We identified 25 RCTs (all placebo-controlled) reporting cognitive outcomes in 46,836 subjects, of which 23 RCTs reported cognitive test results in 29,012 participants. Adverse cognitive outcomes attributable to statins were rarely reported in trials involving cognitively normal or impaired subjects. Furthermore, meta-analysis of cognitive test data (14 studies; 27,643 participants) failed to show significant adverse effects of statins on all tests of cognition in either cognitively normal subjects (standardized mean difference $0.01,95 \%$ confidence interval, CI, -0.01 to $0.03, p=0.42$ ) or Alzheimer's disease subjects (standardized mean difference $-0.05,95 \% \mathrm{CI}-0.19$ to $0.10, p=0.38$ ).

CONCLUSIONS: Statin therapy was not associated with cognitive impairment in RCTs. These results raise

Electronic supplementary material The online version of this article (doi:10.1007/s11606-014-3115-3) contains supplementary material, which is available to authorized users.

Received April 30, 2014

Revised October 3, 2014

Accepted October 27, 2014

Published online January 10, 2015 questions regarding the continued merit of the FDAwarning about potential adverse effects of statins on cognition.

KEY WORDS: statins; systematic review; meta-analysis; randomized control trials; cognition.

J Gen Intern Med 30(3):348-58

DOI: $10.1007 / \mathrm{s} 11606-014-3115-3$

(C) Society of General Internal Medicine 2014

\section{BACKGROUND}

On 28 February 2012, the United States Food and Drug Administration (FDA) issued a new warning for the labeling of statin drugs regarding potential adverse effects on cognition, ${ }^{1}$ based on post-marketing surveillance reports, case reports, observational studies, ${ }^{2-11}$ and randomized controlled trials (RCTs). ${ }^{12-15}$ Post-marketing reports (case series of 60 to 171 individuals) have described ill-defined memory impairment, reversible upon statin discontinuation, ${ }^{1}$ and some observational studies have described adverse cognitive effects that recurred with re-challenge. ${ }^{5,8,16}$

Other reviewers examining RCT and observational study data reported that there is no conclusive evidence that statins cause or contribute to clinically meaningful cognitive impairment, ${ }^{17-19}$ and may actually provide a slight benefit in dementia prevention. ${ }^{20,21}$ A recent systematic review that included RCT data also found no statistically significant effects on cognition, but deemed the evidence to be of low or moderate strength, and called for additional larger and better-designed studies to settle the question. ${ }^{22}$ The FDA advisory selected four of several pertinent RCTs in its review, without being explicit about how these were selected, and when it weighed such information along with other observational data, the advisory came to different conclusions than other reviewers.

Overall, the use of RCT data in quantitative analyses has been limited: meta-analyses of RCT data were performed in only two reports, which included a total of three RCTs each. ${ }^{21,22}$ These reviews used narrow definitions of cognitive outcomes and were not designed to detect signals of adverse cognitive effects of statins. Furthermore, prior reviews often omitted short-term studies that could have captured more immediate side effects on cognition, similar to those noted in 
case reports. ${ }^{5,8}$ To address these limitations, we performed a systematic review and meta-analysis of cognitive test results and adverse event reports from RCTs of statin treatment in cognitively healthy and cognitively impaired individuals in the short as well as the long term.

\section{METHODS}

\section{Study Aims}

We aimed to synthesize current evidence on causal associations between statin therapy and impaired cognition from RCTs. Secondary goals were to determine whether cognitively impaired individuals are particularly vulnerable to any adverse effects on cognition, and whether blood-brain barrier penetrability differentiated among statins as to which were more likely to be associated with adverse cognitive effects.

The search, study selection, and analytic methods of this review were pre-specified in the review protocol provided in Appendix 1, available online. We focused our systematic review on RCTs of statins, because it is more straightforward to make causal claims based on randomized designs.

\section{Data Sources and Searches}

We used four complementary approaches to identify relevant studies (see Fig. 1). First, we recorded all statin RCTs in the inclusion and exclusion lists of three Cochrane reviews. Two examined the effectiveness of statins for the prevention ${ }^{23}$ and treatment of dementia, ${ }^{24}$ covering literature through 2007 and 2008, respectively, and one examined primary prevention of cardiovascular outcomes, ${ }^{25}$ covering literature through 2007. Second, we conducted electronic searches for RCTs of statins crossed with search terms for neurocognitive outcomes through 2008, designed to identify additional RCTs that the Cochrane reviews might have excluded at the abstract level. Third, we used a sensitive search strategy to identify RCTs of statins, irrespective of reported outcomes, between 2008 and December 2012. Finally, we reviewed the full text of all studies included in a recent large network meta-analysis of statins (and their adverse effects), ${ }^{26}$ and perused the reference lists of RCTs identified through our other approaches.

Our electronic database searches covered MEDLINE, EMBASE, and the Cochrane Central registry of trials. The complete search strategies are provided in Appendix 2, available online. We included only articles published in English during full text review. To standardize our application of the screening criteria, all authors completed a pilot round of the same 300 abstracts using Abstrackr software. ${ }^{27}$ After consensus agreement was established, we continued with single screening of abstracts. All articles selected in the abstract screening phase were retrieved and examined in full text for eligibility.

\section{Study Selection}

We sought to identify RCTs that compared statin therapy with or without other lipid-lowering agents versus no statins (standard therapy, no therapy, or placebo), and reported cognitive outcomes in individuals with a baseline diagnosis of normal cognition, or people with abnormal cognition (e.g., Alzheimer's disease, traumatic brain injury, neurofibromatosis). We considered only statins approved for use in the United Sates or Europe (Table 1). We included RCTs with a mean number of participants per arm greater than ten, irrespective of follow-up duration, because we were also interested in shortterm and transient effects. We considered the following outcomes: dichotomous classifications of cognitive impairment (e.g., dementia vs. no dementia); cognition measured by validated scales; and test performance in the cognitive domains of executive function, attention, processing speed, memory, working memory, or global metrics combining the above. We also recorded information on cognition-related adverse events, including memory loss, forgetfulness, amnesia, memory impairment, and confusion.

\section{Data Extraction}

We abstracted the following information from all eligible studies: participant characteristics, study characteristics including objectives, year of publication, sample size, setting, country, funding mechanism, duration of follow-up, randomization method, reporting of dropouts, intervention and comparator details, and cognitive outcomes and harms. For categorical outcomes, we extracted the number experiencing the outcome in each arm. For continuous outcomes, we extracted test scores to calculate the mean differences at end of followup. We used differences in net changes (i.e., differences in the final minus baseline values between groups) only when mean differences of final values could not be calculated. ${ }^{28}$ Nonnumerical data were extracted by a single reviewer, and checked by another. Reviewers experienced in meta-analysis extracted numerical data in duplicate. The analysis data set was uploaded on the Systematic Review Data Repository (http:// ahrq-srdr-prod-347362009.us-east-1.elb.amazonaws.com/); a list of cognitive tests used in the included studies is provided in Appendix 3.

\section{Risk of Bias of Individual Studies}

We examined the following methodological items: adequacy of random sequence generation, allocation concealment, blinding of participants, personnel, and outcome assessors to treatment assignment, completeness of follow-up data (defined as loss to follow-up and treatment discontinuation rates lower than $20 \%$ ), and lack of differential loss to follow-up or discontinuation (defined as Fisher's exact $p$ value $>0.05$ or difference in absolute rates $<5 \%$, across study groups). These 


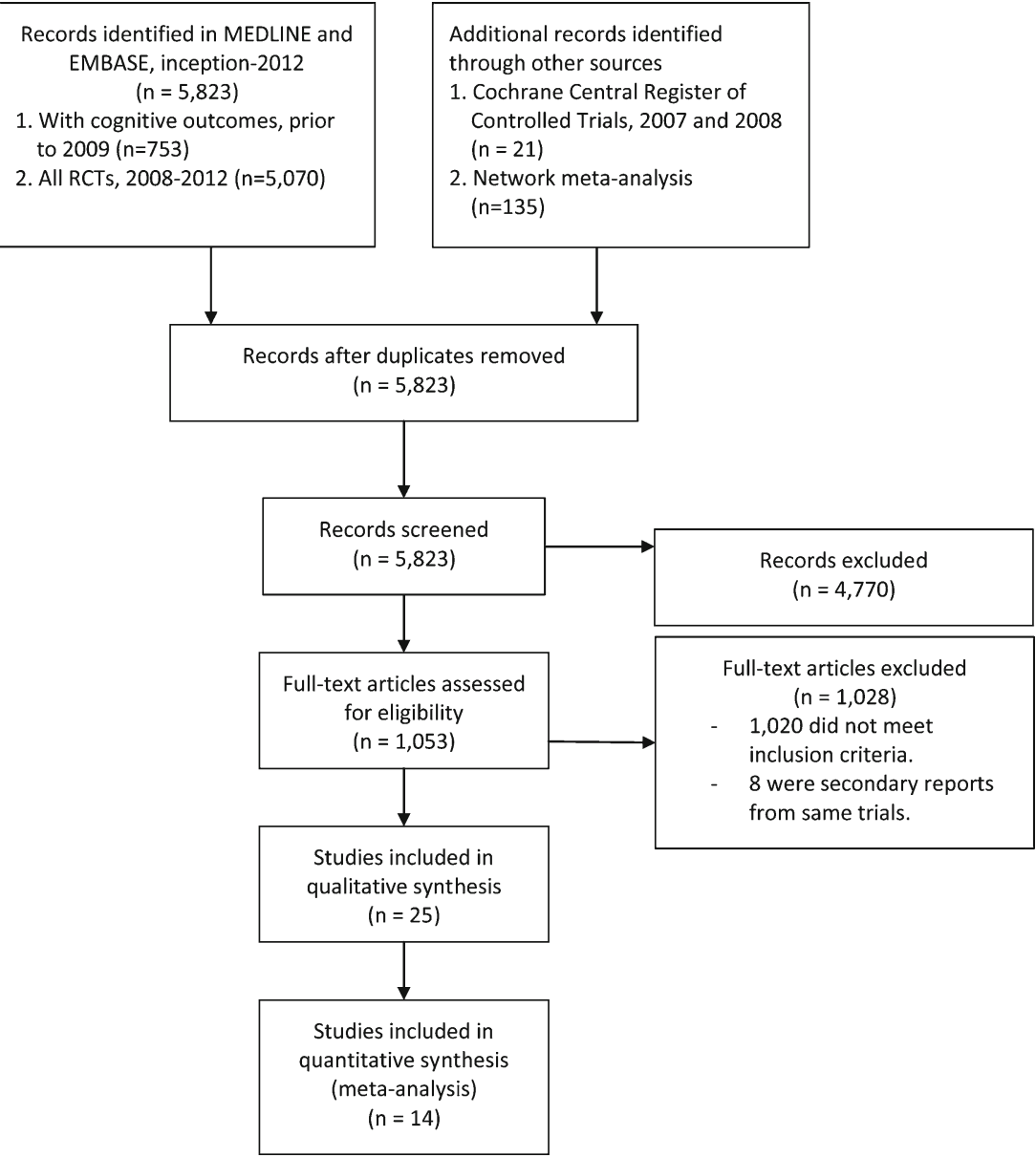

Figure 1 PRISMA flow diagram for literature search and article selection.

correspond to the domains examined by the Cochrane Risk of Bias tool. ${ }^{29}$ Two independent reviewers rated risk of bias as high, low, or unclear. Discrepancies were resolved by consensus.

Table 1. Statin Medications Included in the Systematic Review

\begin{tabular}{|c|c|c|c|}
\hline Statin Name & $\begin{array}{l}\text { Available } \\
\text { Dosage }\end{array}$ & Lipophilicity & $\begin{array}{l}\text { Blood Brain } \\
\text { Barrier } \\
\text { Penetrability }^{35}\end{array}$ \\
\hline $\begin{array}{l}\text { Atorvastatin } \\
\left.\text { (Lipitor }{ }^{\mathbb{B}}\right)\end{array}$ & $10-80 \mathrm{mg}$ & high & low \\
\hline $\begin{array}{l}\text { Fluvastatin } \\
\text { (Lescol and Lescol }\end{array}$ & $20-80 \mathrm{mg}$ & high & high \\
\hline $\begin{array}{l}\text { Lovastatin } \\
\text { (Mevacor; Altoprev }{ }^{\circledR} \text { ) }\end{array}$ & $20-60 \mathrm{mg}$ & high & low \\
\hline $\begin{array}{l}\text { Pravastatin } \\
\left(\text { Pravachol }^{\mathbb{R}}\right)\end{array}$ & $10-80 \mathrm{mg}$ & low & low \\
\hline $\begin{array}{l}\text { Rosuvastatin } \\
\left(\text { Crestor }^{\mathbb{R}}\right)\end{array}$ & $5-40 \mathrm{mg}$ & low & low \\
\hline $\begin{array}{l}\text { Simvastatin } \\
\left(\text { Zocor }^{\mathbb{B}}\right)\end{array}$ & $5-80 \mathrm{mg}$ & high & high \\
\hline $\begin{array}{l}\text { Pitavastatin } \\
\text { (Livalo }{ }^{\mathbb{R}} \text { ) }\end{array}$ & $1-4 \mathrm{mg}$ & high & low \\
\hline
\end{tabular}

\section{Evidence Synthesis}

The results of the systematic review are described narratively. Meta-analyses were performed when information existed from two or more RCTs. Separate meta-analyses were performed for RCTs in cognitively normal subjects, and in patients with Alzheimer's disease (AD). No meta-analyses were done for RCTs in patients with other types of cognitive impairment, because of the small number of available studies for each condition (two or fewer).

Continuous outcomes reported on the same scale (e.g., ADAS-Cog, ${ }^{30} \mathrm{MMSE}^{31}$ ) were summarized as weighted mean differences at the end of follow-up. Continuous outcomes reported on different scales were summarized as standardized mean differences (SMDs), using the Hedges $g$ metric. ${ }^{32}$ SMDs express a difference as a fraction of the pooled standard deviation of the measurements, and allow comparisons across measurements with different instruments, provided that studies come from populations that would have comparable variability in the various measurement scales. While this assumption is often hard to assess, it is frequently used in the psychometrics, education, and psychology literature. SMD 
values of \pm 0.2 imply small to modest differences. ${ }^{33}$ To use all available information and avoid double counting (i.e., to properly account for within-study correlation), we performed meta-analyses using the robust variance estimator proposed by

Table 2. Included Randomized Controlled Trials and Reports of Adverse Cognitive Outcomes

\begin{tabular}{|c|c|c|c|c|c|c|c|}
\hline Author/Trial Year & Population Description & $\begin{array}{l}\text { Age } \\
\text { (years) }\end{array}$ & $\mathbf{N}$ & Design & $\begin{array}{l}\text { Duration } \\
\text { (weeks) }\end{array}$ & Statin Arm (dose) & $\begin{array}{l}\text { Cognitive } \\
\text { Outcomes }\end{array}$ \\
\hline \multicolumn{8}{|c|}{ Populations with normal cognition at baseline } \\
\hline 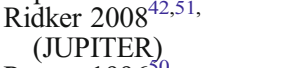 & Healthy with elevated CRP & $60-72$ & 17,802 & $\mathrm{DB}, \mathrm{PC}, \mathrm{P}$ & 92 & $\begin{array}{l}\text { Rosuvastatin } \\
\quad(20 \mathrm{mg})\end{array}$ & Adverse events \\
\hline Posvar $1996^{50}$ & Healthy & $20-46$ & 22 & $\mathrm{DB}, \mathrm{PC}, \mathrm{X}$ & 3 & $\begin{array}{l}\text { Atorvastatin } \\
\quad(0.5-120 \mathrm{mg})\end{array}$ & Adverse events \\
\hline *Muldoon $2004^{14}$ & Healthy & $35-70$ & 283 & $\mathrm{DB}, \mathrm{PC}, \mathrm{P}$ & 24 & $\begin{array}{l}\text { Simvastatin } \\
\quad(10-40 \mathrm{mg})\end{array}$ & Test scores \\
\hline *Muldoon $2000^{13}$ & Healthy & $24-60$ & 209 & $\mathrm{DB}, \mathrm{PC}, \mathrm{P}$ & 24 & $\begin{array}{l}\text { Lovastatin } \\
\quad(20 \mathrm{mg})\end{array}$ & Test scores \\
\hline *Roth $1992^{52}$ & Healthy young men & $18-38$ & 59 & $\mathrm{DB}, \mathrm{PC}, \mathrm{P}$ & 3 & $\begin{array}{l}\text { Lovastatin } \\
\quad(40 \mathrm{mg}) \\
\text { Pravastatin } \\
\quad(40 \mathrm{mg})\end{array}$ & Test scores \\
\hline $\begin{array}{l}{ }^{*} \text { Collins } 2002,{ }^{36} 2004^{40} \\
\text { (HPS) }\end{array}$ & Medically ill & $40-80$ & 20,536 & $\mathrm{DB}, \mathrm{PC}, \mathrm{P}$ & 260 & $\begin{array}{l}\text { Simvastatin } \\
\quad(40 \mathrm{mg})\end{array}$ & $\begin{array}{l}\text { Adverse events } \\
\text { and test scores }\end{array}$ \\
\hline $\begin{array}{l}\text { *Shepherd 2002; }{ }^{56} \\
\text { Trompet 2010 } \\
\text { (PROSPER) }\end{array}$ & Elders at vascular risk & $70-82$ & 5,804 & $\mathrm{DB}, \mathrm{PC}, \mathrm{P}$ & 166 & $\begin{array}{l}\text { Pravastatin } \\
\quad(40 \mathrm{mg})\end{array}$ & Test scores \\
\hline *Santanello $1997^{55}$ & Healthy elders & $>65$ & 431 & $\mathrm{DB}, \mathrm{PC}, \mathrm{P}$ & 24 & $\begin{array}{l}\text { Lovastatin } \\
\quad(20-40 \mathrm{mg})\end{array}$ & Test scores \\
\hline *Gibellato $2001^{44}$ & Military air crew & $23-50$ & 80 & $\mathrm{DB}, \mathrm{PC}, \mathrm{P}$ & 4 & $\begin{array}{l}\text { Lovastatin } \\
\quad(40 \mathrm{mg}) \\
\text { Pravastatin } \\
\quad(40 \mathrm{mg})\end{array}$ & Test scores \\
\hline *Summers $2007^{62}$ & Chronic renal disease & $25-83$ & 57 & $\mathrm{DB}, \mathrm{PC}, \mathrm{P}$ & 36 & $\begin{array}{l}\text { Atorvastatin } \\
(10 \mathrm{mg})\end{array}$ & Test scores \\
\hline${ }^{*}$ Carlson $2002^{38}$ & Healthy & $71-86$ & 41 & $\mathrm{DB}, \mathrm{PC}, \mathrm{X}$ & 24 & $\begin{array}{l}\text { Pravastatin } \\
\quad(20 \mathrm{mg})\end{array}$ & Test scores \\
\hline *Cutler $1995^{41}$ & Healthy middle age & $40-60$ & 36 & $\mathrm{DB}, \mathrm{PC}, \mathrm{X}$ & 4 & $\begin{array}{l}\text { Pravastatin } \\
\quad(40 \mathrm{mg}) \\
\text { Simvastatin } \\
\text { (20 mg) }\end{array}$ & Test scores \\
\hline *Gengo $1995^{43}$ & Healthy middle age & $40-60$ & 36 & $\mathrm{DB}, \mathrm{PC}, \mathrm{P}$ & 4 & $\begin{array}{l}\text { Lovastatin } \\
(40 \mathrm{mg}) \\
\text { Pravastatin } \\
(40 \mathrm{mg})\end{array}$ & Test scores \\
\hline *Harrison $1994^{45}$ & Healthy young & $20-32$ & 25 & $\mathrm{DB}, \mathrm{PC}, \mathrm{X}$ & 4 & $\begin{array}{l}\text { Pravastatin } \\
\quad(40 \mathrm{mg}) \\
\text { Simvastatin } \\
\quad(40 \mathrm{mg})\end{array}$ & Test scores \\
\hline Kostis $1994^{48}$ & Healthy men & $36-65$ & 22 & $\mathrm{DB}, \mathrm{PC}, \mathrm{X}$ & 6 & $\begin{array}{l}\text { Lovastatin } \\
\quad(40 \mathrm{mg}) \\
\text { Pravastatin } \\
(40 \mathrm{mg})\end{array}$ & Test scores \\
\hline${ }^{*}$ Carlson $2008^{38}$ & Children of AD parents & $40-65$ & 57 & $\mathrm{DB}, \mathrm{PC}, \mathrm{P}$ & 16 & $\begin{array}{l}\text { Simvastatin } \\
\quad(40 \mathrm{mg})\end{array}$ & Test scores \\
\hline *Tendolkar $2011^{64}$ & Elders/ atrial fibrillation & mean 74 & 34 & $\mathrm{DB}, \mathrm{PC}, \mathrm{P}$ & 52 & $\begin{array}{l}\text { Atorvastatin } \\
\text { (40 mg) }\end{array}$ & Test scores \\
\hline Berk-Planken $2002^{37}$ & Diabetics & $45-75$ & 30 & $\mathrm{DB}, \mathrm{PC}, \mathrm{P}$ & 30 & $\begin{array}{l}\text { Atorvastatin } \\
\quad(10-80 \mathrm{mg})\end{array}$ & Test scores \\
\hline \multicolumn{8}{|c|}{ Populations with impaired cognition at baseline } \\
\hline Feldman $2010^{12}$ & Alzheimer's disease & $\begin{array}{l}\text { mean } \\
73\end{array}$ & 640 & $\mathrm{DB}, \mathrm{PC}, \mathrm{P}$ & 72 & $\begin{array}{l}\text { Atorvastatin } \\
\quad(80 \mathrm{mg})\end{array}$ & Test scores \\
\hline Sano $2011^{54}$ & Alzheimer's disease & mean 75 & 406 & $\mathrm{DB}, \mathrm{PC}, \mathrm{P}$ & 72 & $\begin{array}{l}\text { Simvastatin } \\
\quad(40 \mathrm{mg})\end{array}$ & Test scores \\
\hline Sparks $2005^{58}$ & Alzheimer's disease & mean 78 & 63 & $\mathrm{DB}, \mathrm{PC}, \mathrm{P}$ & 48 & $\begin{array}{l}\text { Atorvastatin } \\
\quad(80 \mathrm{mg})\end{array}$ & Test scores \\
\hline Simons $2002^{57}$ & Alzheimer's disease & mean 68 & 44 & $\mathrm{DB}, \mathrm{PC}, \mathrm{P}$ & 26 & $\begin{array}{l}\text { Simvastatin } \\
\quad(80 \mathrm{mg})\end{array}$ & Test scores \\
\hline Krab $2008^{49}$ & Neuro-fibromatosis 1 & $8-16$ & 62 & $\mathrm{DB}, \mathrm{PC}, \mathrm{P}$ & 12 & $\begin{array}{l}\text { Simvastatin } \\
\quad(20-40 \mathrm{mg})\end{array}$ & Test scores \\
\hline Sanchez-Aguilar $2013^{53}$ & Traumatic brain injury & $19-32$ & 36 & $\mathrm{DB}, \mathrm{PC}, \mathrm{P}$ & 2 & $\begin{array}{l}\text { Rosuvastatin } \\
\text { (20 mg) }\end{array}$ & Test scores \\
\hline Tapia $2008^{63}$ & Traumatic brain injury & $16-50$ & 21 & $\mathrm{DB}, \mathrm{PC}, \mathrm{P}$ & 2 & $\begin{array}{l}\text { Rosuvastatin } \\
\quad(20 \mathrm{mg})\end{array}$ & Test scores \\
\hline
\end{tabular}

* Studies included in meta-analysis of cognitively normal individuals. The remaining two studies did not report adequate data for quantitative synthesis, but reported that no statistically significant differences were observed

DB double-blind trial; HPS Heart Protection Study; JUPITER Justification for the Use of Statins in Primary Prevention: An Intervention Trial Evaluating Rosuvastatin; PC placebo-controlled trial; P parallel group trial; PROSPER Prospective Study of Pravastatin in the Elderly at Risk; $X$ cross-over trial 
Hedges. $^{34}$ This approach adjusts the variance of the metaanalysis summary to account for model mis-specification.

All meta-analyses were done with a random effects model, because substantial between-study diversity was expected a priori. Models were fit with weighted leastsquares methods. We assessed the extent of betweenstudy heterogeneity using the estimated between-study standard deviation (i.e., $\tau$, the square root of the between-study variance). Larger estimated $\tau$ values indicate the presence of greater heterogeneity.

\section{Assessment of Heterogeneity and Meta-Regression Analyses}

Random effects meta-regression with weighted least squares was used to investigate the contribution of the degree of blood brain barrier penetrability to between-study differences for cognitive effects. For this analysis, statins were divided into two categories, according to higher (simvastatin only, since no studies with fluvastatin are included) vs. lower (all others) propensity to cross the blood-brain barrier. ${ }^{35}$ Additional analyses were performed for study duration, study sample size, and risk of bias items, when the data allowed (i.e., when a characteristic varied across studies and six or more studies were available for analysis).

\section{RESULTS}

\section{Study Characteristics}

Figure 1 shows the literature search and article selection flow chart. We screened 5,823 citations and evaluated the full text of 1,827 articles, and included $25 \mathrm{RCTs}$ (Table 2) reported in 33 publications. ${ }^{12-15,36-64}$ All had placebo rather than standard care comparators. RCTs (reported in 23 publications ${ }^{13-15,36-48,50-52,55,56,62,64}$ ) enrolled subjects with normal cognition at baseline, four RCTs (reported in seven publications ${ }^{12,54,57-61}$ ) enrolled patients with AD, and three RCTs enrolled other cognitively impaired subjects (traumatic brain injury, ${ }^{53,63}$ and neurofibromatosis type $1^{49}$ ). Simvastatin (eight trials), pravastatin (eight trials), and lovastatin (six trials) were most frequently utilized in the statin RCTs. There were no RCTs that assessed neurocognitive outcomes for fluvastatin and pitavastatin.

Overall, we found that studies were at low to moderate risk of bias. Procedures for randomized sequence generation and allocation concealment were deemed adequate in only 13 $(52 \%)$ and nine $(36 \%)$ studies, respectively, of the 25 studies included in the review. In contrast, blinding of participants, personnel, and outcome assessors was adequate in the majority of studies ( $>90 \%$ for all three types of blinding). Losses-

Table 3. Assessment of Study Validity

\begin{tabular}{|c|c|c|c|c|c|c|c|}
\hline $\begin{array}{l}\text { Author/Trial } \\
\text { Year }\end{array}$ & $\begin{array}{l}\text { Random } \\
\text { sequence } \\
\text { generation }\end{array}$ & $\begin{array}{l}\text { Allocation } \\
\text { concealment }\end{array}$ & $\begin{array}{l}\text { Blinding of } \\
\text { participants }\end{array}$ & $\begin{array}{l}\text { Blinding of } \\
\text { personnel }\end{array}$ & $\begin{array}{l}\text { Blinding of } \\
\text { outcome } \\
\text { assessors }\end{array}$ & $\begin{array}{l}\text { Complete outcome data } \\
\text { (<20\% loss-to-follow-up } \\
\text { and study discontinuations) }\end{array}$ & $\begin{array}{l}\text { Lack of } \\
\text { differential } \\
\text { dropout? }\end{array}$ \\
\hline \multicolumn{8}{|c|}{ Cognitively normal individuals } \\
\hline $\begin{array}{l}\text { Ridker 2008 } \\
\text { (JUPITER) }\end{array}$ & Yes & Yes & Yes & Yes & Yes & Yes & Yes \\
\hline Posvar $1996^{50}$ & Unclear & Unclear & No & No & No & Yes & Yes \\
\hline Muldoon $2004^{14}$ & Yes & Unclear & Yes & Yes & Yes & Yes & Yes \\
\hline Muldoon $2000^{13}$ & Unclear & Unclear & Yes & Yes & Yes & Yes & Unclear \\
\hline Roth $1992^{52}$ & Unclear & Unclear & Yes & Yes & Yes & Yes & Unclear \\
\hline $\begin{array}{l}\text { Collins } \\
2002,362004^{40} \\
\text { (HPS) }\end{array}$ & Yes & Yes & Yes & Yes & Yes & Yes & Yes \\
\hline $\begin{array}{l}\text { * Shepherd 2002.56 } \\
\text { Trompet 2010 } \\
\text { (PROSPER) }\end{array}$ & Yes & Yes & Yes & Yes & Yes & No & Yes \\
\hline Santanello $1997^{55}$ & Unclear & Unclear & Yes & Yes & Yes & Yes & Yes \\
\hline Gibellato $2001^{44}$ & Unclear & Unclear & Yes & Yes & Yes & Yes & Yes \\
\hline Summers $2007^{62}$ & Unclear & Unclear & Yes & Yes & Yes & No & Unclear \\
\hline Carlson $2002^{38}$ & Unclear & Unclear & Yes & Yes & Yes & Yes & Unclear \\
\hline Cutler $1995^{41}$ & Unclear & Unclear & Yes & Yes & Yes & Yes & Yes \\
\hline Gengo $1995^{43}$ & Unclear & Unclear & Yes & Yes & Yes & Yes & Yes \\
\hline Harrison $1994^{45}$ & Unclear & Unclear & Yes & No & Yes & Yes & Unclear \\
\hline Kostis $1994^{48}$ & Unclear & Unclear & Yes & Yes & Yes & Yes & Yes \\
\hline Carlson $2008^{38}$ & Yes & Unclear & Yes & Yes & Yes & Yes & Yes \\
\hline Tendolkar $2011^{64}$ & Yes & Unclear & Yes & Yes & Yes & Yes & Yes \\
\hline Berk-Planken $2002^{37}$ & Unclear & Unclear & Yes & Yes & Yes & Yes & Unclear \\
\hline \multicolumn{8}{|c|}{ Patients with baseline cognitive impairment } \\
\hline Feldman $2010^{12}$ & Yes & Yes & Yes & Yes & Yes & No & No \\
\hline Sano $2011^{54}$ & Yes & Yes & Yes & Yes & Yes & Yes & Yes \\
\hline Sparks $2005^{58}$ & Yes & Yes & Yes & Yes & Yes & No & Yes \\
\hline Simons $2002^{57}$ & Yes & Yes & Yes & Yes & Yes & Yes & Yes \\
\hline Krab $2008^{49}$ & Yes & Yes & Yes & Yes & Yes & Yes & Yes \\
\hline $\begin{array}{l}\text { Sanchez-Aguilar } \\
2013^{53}\end{array}$ & Yes & Unclear & Yes & Yes & Yes & Yes & Yes \\
\hline Tapia $2008^{63}$ & Yes & Yes & Yes & Yes & Yes & Yes & Yes \\
\hline
\end{tabular}

AD Alzheimer's disease; HPS Heart Protection Study; JUPITER Justification for the Use of Statins in Primary Prevention: An Intervention Trial Evaluating Rosuvastatin; PROSPER Prospective Study of Pravastatin in the Elderly at Risk 
Table 4. Meta-Analysis of Treatment Effect of Statins on Global Cognition and Cognitive Domains According to Subject Characteristics

\begin{tabular}{|c|c|c|c|c|c|c|}
\hline Domain or Scale & Sample Size & Number of Studies & $\begin{array}{l}\text { Number of Effect } \\
\text { Sizes Synthesized }\end{array}$ & Summary Effect Size* & $P$ value & $\begin{array}{l}\text { Square root of between- } \\
\text { study variance }(\tau)\end{array}$ \\
\hline \multicolumn{7}{|c|}{ Among populations with normal cognition at baseline } \\
\hline All effects & 27,643 & 14 & 131 & $0.01(-0.01,0.03)$ & 0.423 & 0.046 \\
\hline Global & 26,515 & 5 & 23 & $-0.02(-0.04,0.01)$ & 0.207 & 0.018 \\
\hline Attention & 732 & 7 & 36 & $0.10(-0.17,0.37)$ & 0.402 & 0.254 \\
\hline Executive & 26,926 & 7 & 19 & $0.04(-0.02,0.09)$ & 0.179 & 0.045 \\
\hline Memory & 26,850 & 8 & 18 & $0.00(-0.01,0.02)$ & 0.380 & 0.005 \\
\hline Processing speed & 6,630 & 10 & 16 & $0.01(-0.13,0.15)$ & 0.879 & 0.149 \\
\hline Working memory & 83 & 3 & 6 & $-0.35(-1.24,0.55)$ & 0.236 & 0.244 \\
\hline \multicolumn{7}{|c|}{ Among populations with Alzheimer's disease } \\
\hline All effects & 935 & 4 & 10 & $-0.05(-0.19,0.10)$ & 0.381 & 0.185 \\
\hline ADAS-Cog & 926 & 4 & 4 & $0.97(-2.21,4.16)^{* *}$ & 0.403 & 1.231 \\
\hline MMSE & 945 & 4 & 4 & $-0.67(-1.21,-0.13)^{* *}$ & 0.029 & 0.329 \\
\hline
\end{tabular}

* Standardized mean differences, unless otherwise noted

** Unstandardized mean differences

ADAS-cog Alzheimer's Disease Assessment Scale, cognition; MMSE Mini-Mental State Examination

to-follow-up and dropouts totaled less than $20 \%$ of the enrolled population in $21(84 \%)$ of the studies. Differential dropout between compared groups was deemed significant or impossible to assess in seven studies (28\%). Study-level risk-of-bias information is presented in Table 3.

\section{Studies of Cognitively Normal Participants}

The 18 RCTs of cognitively normal individuals included heterogeneous populations. Twelve RCTs (1,301 patients) enrolled generally healthy participants (there was no index medical condition), while six (44,263 patients) enrolled patients at elevated risk for cardiovascular disease. Nine out of 18 RCTs also included patients aged 65 years or older, with the remainder including younger patients.
Dementia, Confusion, and Other Cognitive Adverse Events. Development of dementia, confusion and other cognitive adverse events was reported in three out of 18 RCTs in cognitively healthy participants. The JUPITER trial [Justification for the Use of Statins in Primary Prevention: An Intervention Trial Evaluating Rosuvastatin] $(n=17,802)^{42}$ comparing rosuvastatin vs. placebo reported no statistically significant differences for the incidence of dementia (12 vs. nine patients, respectively), confusion (18 vs. four patients, respectively), or adverse nervous system events (69 vs. 76 patients, respectively). The absolute event rate was less than $1 \%$ for all these outcomes. The HPS trial [Heart Protection Study] $(n=20,536)^{36}$ comparing simvastatin versus placebo found no statistically significant differences in the incidence of dementia (31 vs. 31 patients,

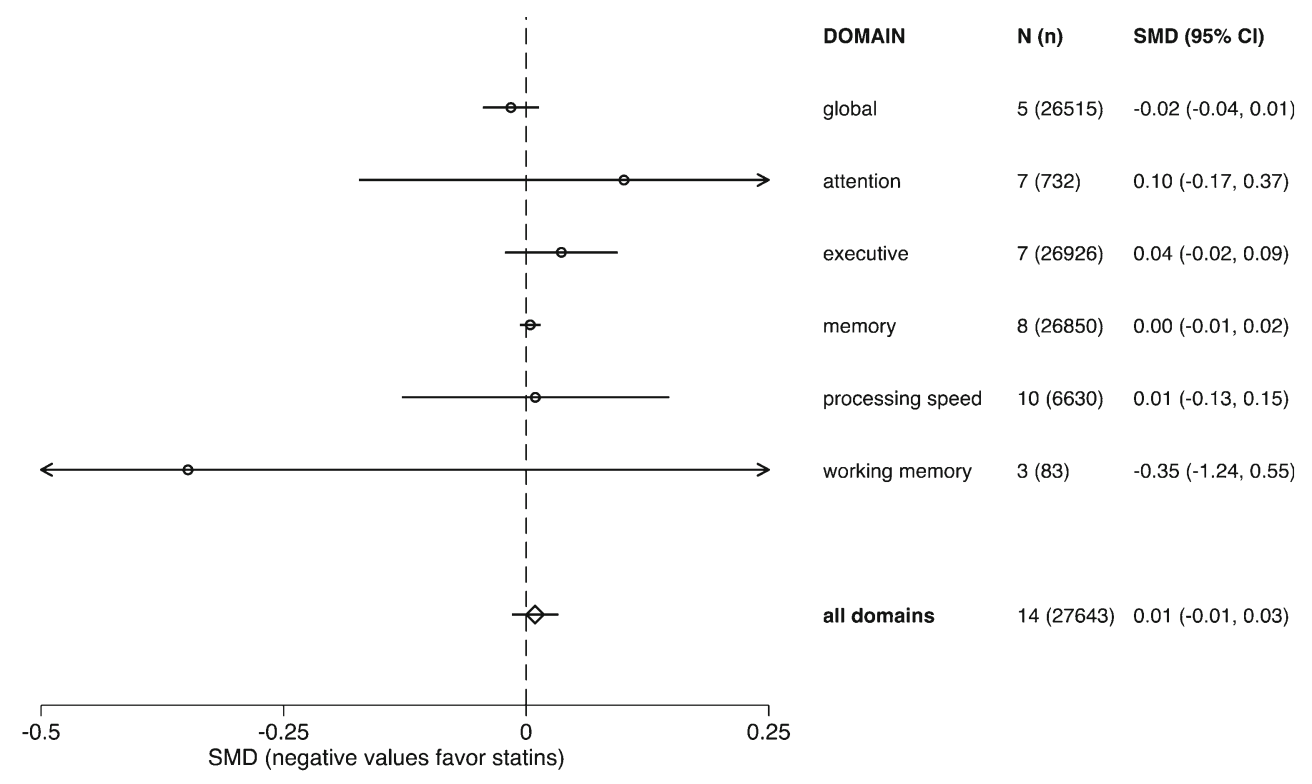

Negative SMD values indicate better cognition in statin compared to no statin arms.

$\mathrm{Cl}=$ confidence interval; $\mathrm{N}=$ number of studies; $\mathrm{n}=$ number of patients included in analyses; $\mathrm{SMD}=$ standardized mean difference.

Figure 2 Forest plot of treatment effect of statins on global cognition and cognitive domains in cognitively normal subjects. 
espectively) or the proportion of patients classified as cognitively impaired by telephone-administered cognitive assessment at end of study (2,433 or $24 \%$ vs. 2,485 or $24 \%$, respectively). Finally, a phase 1 dose-escalation study of atorvastatin vs. placebo in 22 healthy young adults reported dose-limiting central nervous system adverse effects in one person receiving a solution of $120 \mathrm{mg}$ of atorvastatin, including mild, transient restlessness, euphoria, and mental confusion. ${ }^{50}$

Cognitive Test Outcomes. Cognitive test outcomes were assessed in 16 RCTs $(27,693$ patients total). Various tests were employed. "Global" measures included test scores that covered multiple cognitive domains, while "all effects" in Table 4 refers to a calculated overall measure of cognition, combining SMDs across all cognitive domains. Two of the 16 studies found no statistically significant effect of statins on cognitive tests, but did not report adequate data for inclusion in meta-analyses. Meta-analyses of the remaining 14 studies found no statistically significant differences between statin and no statin groups (Table 4 and Fig. 2), for the global, attention, executive, memory, processing speed and working

Table 5. Meta-Analysis of Treatment Effect (Standardized Mean Difference) of Statins on Cognition by Statin Characteristics in Populations with Normal Cognition at Baseline

\begin{tabular}{|c|c|c|c|c|c|c|}
\hline Covariate & $\begin{array}{l}\text { Domain or } \\
\text { Scale }\end{array}$ & $\begin{array}{l}\text { Covariate } \\
\text { value }\end{array}$ & $\begin{array}{l}\text { Number } \\
\text { of Studies }\end{array}$ & $\begin{array}{l}\text { Summary Effect } \\
\text { Size (SMD) }\end{array}$ & $\begin{array}{l}\text { Difference of Effect } \\
\text { Size Across Subgroups }\end{array}$ & $P$ value \\
\hline \multirow{10}{*}{$\begin{array}{l}\text { BBB penetrant } \\
\text { (simvastatin) }\end{array}$} & \multirow[t]{2}{*}{ All effects } & Simvastatin & 5 & $0.018(-0.029,0.065)$ & $0.003(-0.105,0.111)$ & 0.959 \\
\hline & & All other statins & 9 & $0.015(-0.082,0.112)$ & & \\
\hline & \multirow[t]{2}{*}{ Attention } & Simvastatin & 3 & $0.273(-0.387,0.933)$ & $0.297(-0.378,0.972)$ & 0.309 \\
\hline & & All other statins & 4 & $-0.024(-0.164,0.116)$ & & \\
\hline & \multirow[t]{2}{*}{ Executive } & Simvastatin & 3 & $0.057(-0.206,0.319)$ & $-0.005(-0.293,0.284)$ & 0.969 \\
\hline & & All other statins & 4 & $0.061(-0.057,0.180)$ & & \\
\hline & \multirow[t]{2}{*}{ Memory } & Simvastatin & 4 & $0.017(-0.016,0.051)$ & $0.044(-0.027,0.114)$ & 0.179 \\
\hline & & All other statins & 4 & $-0.027(-0.088,0.035)$ & & \\
\hline & \multirow[t]{2}{*}{ Processing speed } & Simvastatin & 3 & $-0.032(-0.083,0.018)$ & $-0.054(-0.270,0.161)$ & 0.578 \\
\hline & & All other statins & 7 & $0.022(-0.188,0.232)$ & & \\
\hline \multirow{10}{*}{$\begin{array}{l}\text { Random sequence } \\
\text { generation }\end{array}$} & \multirow[t]{2}{*}{ All effects } & Low risk of bias & 5 & $0.000(-0.020,0.020)$ & $-0.053(-0.160,0.054)$ & 0.3 \\
\hline & & High risk of bias & 9 & $0.053(-0.052,0.158)$ & & \\
\hline & \multirow[t]{2}{*}{ Attention } & Low risk of bias & 2 & $0.305(-0.622,1.232)$ & $0.288(-0.653,1.228)$ & 0.468 \\
\hline & & High risk of bias & 5 & $0.017(-0.145,0.180)$ & & \\
\hline & \multirow[t]{2}{*}{ Executive } & Low risk of bias & 4 & $0.031(-0.035,0.097)$ & $-0.078(-0.285,0.129)$ & 0.377 \\
\hline & & High risk of bias & 3 & $0.109(-0.088,0.306)$ & & \\
\hline & \multirow[t]{2}{*}{ Memory } & Low risk of bias & 5 & $0.003(-0.012,0.018)$ & $0.076(-0.049,0.202)$ & 0.187 \\
\hline & & High risk of bias & 3 & $-0.073(-0.197,0.051)$ & & \\
\hline & \multirow{2}{*}{ Processing speed } & Low risk of bias & 3 & $-0.153(-0.544,0.237)$ & $-0.253(-0.694,0.188)$ & 0.222 \\
\hline & & High risk of bias & 7 & $0.100(-0.105,0.304)$ & & \\
\hline \multirow{6}{*}{$\begin{array}{l}\text { Allocation } \\
\text { concealment }\end{array}$} & \multirow[t]{2}{*}{ All effects } & Low risk of bias & 2 & $0.002(-0.002,0.005)$ & $-0.031(-0.123,0.061)$ & 0.477 \\
\hline & & High risk of bias & 12 & $0.033(-0.059,0.125)$ & & \\
\hline & \multirow[t]{2}{*}{ Executive } & Low risk of bias & 2 & $0.018(-0.012,0.048)$ & $-0.125(-0.361,0.111)$ & 0.231 \\
\hline & & High risk of bias & 5 & $0.143(-0.091,0.377)$ & & \\
\hline & \multirow[t]{2}{*}{ Memory } & Low risk of bias & 2 & $0.003(-0.012,0.017)$ & $0.020(-0.195,0.234)$ & 0.829 \\
\hline & & High risk of bias & 6 & $-0.017(-0.231,0.197)$ & & \\
\hline \multirow{8}{*}{$\begin{array}{l}\text { Dropouts and loss to } \\
\text { follow-up }<20 \%\end{array}$} & \multirow[t]{2}{*}{ All effects } & Low risk of bias & 9 & $-0.003(-0.020,0.015)$ & $-0.146(-0.294,0.003)$ & 0.054 \\
\hline & & High risk of bias & 5 & $0.143(-0.004,0.290)$ & & \\
\hline & \multirow[t]{2}{*}{ Attention } & Low risk of bias & 5 & $0.133(-0.336,0.602)$ & $0.079(-0.396,0.555)$ & 0.686 \\
\hline & & High risk of bias & 2 & $0.054(-0.029,0.136)$ & & \\
\hline & Executive & Low risk of bias & 5 & $0.031(-0.033,0.095)$ & $-0.094(-0.319,0.130)$ & 0.33 \\
\hline & & High risk of bias & 2 & $0.125(-0.090,0.340)$ & & \\
\hline & Processing speed & Low risk of bias & 6 & $-0.069(-0.213,0.075)$ & $-0.315(-0.642,0.013)$ & 0.057 \\
\hline & & High risk of bias & 4 & $0.246(-0.048,0.539)$ & & \\
\hline Study duration & All effects & $>24$ weeks & 3 & $-0.005(-0.023,0.014)$ & $-0.058(-0.140,0.025)$ & 0.153 \\
\hline & & $\leq 24$ weeks & 11 & $0.053(-0.027,0.133)$ & & \\
\hline & Executive & $>24$ weeks & 2 & $0.018(-0.012,0.048)$ & $-0.125(-0.361,0.111)$ & 0.231 \\
\hline & & $\leq 24$ weeks & 5 & $0.143(-0.091,0.377)$ & & \\
\hline & Memory & $>24$ weeks & 3 & $0.001(-0.016,0.018)$ & $-0.025(-0.165,0.114)$ & 0.671 \\
\hline & & $\leq 24$ weeks & 5 & $0.026(-0.112,0.164)$ & & \\
\hline & Processing speed & $>24$ weeks & 2 & $-0.193(-0.750,0.364)$ & $-0.275(-0.862,0.311)$ & 0.311 \\
\hline & & $\leq 24$ weeks & 8 & $0.082(-0.103,0.267)$ & & \\
\hline Sample size & All effects & $>100$ participants & 5 & $0.011(-0.010,0.033)$ & $0.015(-0.181,0.211)$ & 0.87 \\
\hline & & $\leq 100$ participants & 9 & $-0.004(-0.198,0.191)$ & & \\
\hline & Attention & $>100$ participants & 2 & $-0.014(-0.090,0.063)$ & $-0.198(-0.701,0.305)$ & 0.358 \\
\hline & & $\leq 100$ participants & 5 & $0.185(-0.313,0.682)$ & & \\
\hline & Executive & $>100$ participants & 4 & $0.043(-0.032,0.119)$ & $0.165(-0.104,0.435)$ & 0.176 \\
\hline & & $\leq 100$ participants & 3 & $-0.122(-0.381,0.137)$ & & \\
\hline & Memory & $>100$ participants & 3 & $0.006(-0.004,0.016)$ & $0.150(-0.079,0.379)$ & 0.161 \\
\hline & & $\leq 100$ participants & 5 & $-0.144(-0.373,0.085)$ & & \\
\hline & Processing speed & $>100$ participants & 2 & $-0.006(-0.022,0.011)$ & $-0.027(-0.326,0.272)$ & 0.843 \\
\hline & & $\leq 100$ participants & 8 & $0.021(-0.278,0.320)$ & & \\
\hline
\end{tabular}

Negative SMDs imply that outcomes are favorable in statin compared to non-statin groups. Subgroup and meta-regression analyses were performed when $\geq 6$ studies were available for an outcome and $\geq 2$ were available within each subgroup

$B B B$ blood brain barrier; SMD standardized mean difference 
memory domains. Summary effect sizes ranged between -0.35 and 0.10 . With the exception of the working memory domain, which was rather imprecise, $95 \%$ CIs excluded small to moderate effect sizes (e.g., effect sizes as large as 0.20 in either direction, and often much smaller, were not within the $95 \%$ CIs of summary estimates). Across all cognitive domains, we found little evidence of an adverse impact of statins on cognitive outcomes: $\mathrm{SMD}=0.01 ; 95 \% \mathrm{CI}-0.01$ to 0.03 ).

Between-study heterogeneity was low overall and for specific cognitive domains, with the exception of attention and working memory, where heterogeneity was more pronounced. Subgroup analyses according to blood-brain barrier penetrating statins suggested no differences in effect size. Similarly, risk-of-bias items for which meta-regression analyses were possible did not reveal any associations with the estimated treatment effects. Results from regression analyses are shown in Table 5.

Cognitively Impaired Populations. The four RCTs in 1,153 patients with an established diagnosis of $\mathrm{AD}$ included primarily older adults (mean ages above 68 years). The two largest RCTs that contributed the majority of patients $(1,046 / 1,153$ or $91 \%)$ were deemed to have low risk of bias. $^{12,54}$ Table 3 summarizes study-level assessments for risk of bias. In a random effects meta-analysis, the weighted mean differences for the ADAS-cog and MMSE instruments were higher in the statin compared to no statin arms, suggesting a trend toward benefit, but the $95 \%$ confidence intervals were broad (see Table 4). Based on the confidence interval boundaries, the meta-analysis practically excludes a clinically important difference in ADAS-cog, which is approximately three to four points. ${ }^{65}$ Although the summary result for the MMSE suggested the presence of a beneficial statin effect $(p<0.05)$, the association was no longer significant after adjustment for multiple comparisons or when analyses were repeated using net changes (instead of differences in final values).

Finally, the effect of 10 days of statin treatment on global cognitive function in subjects with acute traumatic brain injury was investigated in two small RCTs, from the same research team. One showed no statistically significant cognitive benefit over 6 months, ${ }^{53}$ and the other showed positive benefit over 4 months. ${ }^{63}$ A small trial of 62 children with neurofibromatosis type 1 , expected to have a high prevalence of learning disability, showed no significant effects for statin treatment. ${ }^{49}$

\section{DISCUSSION}

The FDA warning about potential adverse cognitive effects of statins has important public health implications; therefore, a thorough assessment of the effects of statins should rely on information from all available RCTs. To this end, we conducted meta-analyses designed to detect signals for adverse neurocognitive outcomes. We found no statistically significant effects of statin treatment on cognition. If anything, for cognitive domains such as memory, where the cumulative sample size is substantial, confidence intervals of the summary effect exclude even very small differences (e.g., SMDs of 0.05) in either direction. By contrast, when data was limited, such as for the working memory domain, confidence intervals were wide, and we could not exclude large beneficial or harmful effects. Between-study heterogeneity was higher for attention and working memory than other domains. In terms of an overall signal however, our analyses of SMDs for all cognitive outcomes do not confirm the signal detected from spontaneous adverse event reports. We also sought to explore the impact of study-level characteristics on the association of statin treatment with cognitive outcomes. We found no significant effects or modification of effects within or across neurocognitive domains such as memory or attention, by whether the drug penetrates the blood-brain barrier or not, study duration, sample size, location, or cognitive health status.

Our results suggest that the FDA warning about potential adverse effects of statins on cognition merits re-evaluation. The risks for cognition, if any, are likely outweighed by the beneficial effects of adherence to statin therapy on cardiovascular and cerebrovascular disease. ${ }^{66}$ In addition, the reasons for the discordance between trial results and reports from the Adverse Events Reporting System (or published case series) should be explored further. One explanation might be that cognitive adverse effects are more likely to occur at high statin dosages. Only five RCTs employed doses at the upper limit of current guidelines, so we cannot exclude possible adverse effects from high dosage. However, three of the four AD trials did use high doses, and significant negative effects were not seen in this particularly vulnerable population. In one study included in this review, a patient experienced confusion after receiving $120 \mathrm{mg}$ of atorvastatin, a dose that is $40 \mathrm{mg}$ higher than the recommended daily dose for treating hyperlipidemia. ${ }^{50}$ Neurotoxic effects associated with high dose statins have been reported in laboratory animals ${ }^{67-69}$ and a phase 1 study in humans. ${ }^{70}$ In the less controlled environment of home use, it is possible that some individuals reporting cognitive adverse events may have unintentionally overdosed. In addition, other factors (grapefruit juice ingestion, ${ }^{71,72}$ drug interactions, ${ }^{73,74}$ or constitutional differences in drug metabolism) can result in higher-than-expected statin plasma levels and subsequent adverse effects.

Compared to previous reviews on this topic, our approach is more comprehensive (it includes data from over $20 \mathrm{RCTs}$ ), and employs well-studied analysis methods. A previous systematic review summarized evidence for each cognitive test separately, and employed a "vote counting" approach for detecting a signal in RCT data. ${ }^{22}$ Evaluating each cognitive test in isolation does not allow learning across trials that assess similar cognitive outcomes measured on different scales, and does not facilitate signal detection for adverse events. Furthermore, vote counting also fails to account for the magnitude of observed effects or their precision. 
To detect signals of small magnitude, one must maximize the use of the available information. We made methodological choices to address three challenges to using all available information. The first challenge was that the RCTs measured a variety of cognitive outcomes. For example, among the $18 \mathrm{RCTs}$ in people with normal cognition, any instrument was used in three RCTs at most, and, often, in only one. We opted to use standardized effect sizes to synthesize information across distinct instruments measuring the same construct.

The second challenge was that many RCTs used two or more cognitive tests from the same cognitive domain. Such outcomes are correlated, because they are measured in the same patients, and are in some sense "partially redundant." At the same time, each provides potentially useful information. Instead of arbitrarily choosing one of several tests from a RCT, we synthesized all of them and accounted for their correlations by calculating robust variance estimates. ${ }^{34}$

The third challenge was integrating results from the various cognitive domains for detecting an overall signal regarding the cognitive effects of statins. To this end, we calculated an omnibus overall measure of cognition across all cognitive domains. This measure does not have a straightforward interpretation, in that it does not stand for a particular outcome or cognitive domain. However, it does address the high level question of whether a "signal" with adequate statistical power exists for potential cognitive effects of statin treatments.

Our approach has limitations. First, publication bias and selective outcome reporting threaten the validity of all metaanalyses. When these biases operate, statistically significant findings are more likely to be reported in full, compared to findings of no difference and statistically nonsignificant results. Second, standardized effect sizes, especially when combining across cognition domains, are difficult to interpret. However, standardized effects may well suffice for detecting the presence or absence of a signal. In our case, the summary for the overall outcome was practically zero, and the confidence intervals were narrow enough to exclude important signals. Third, we interpreted the metaanalysis for overall cognition to suggest that statins do not have important effects on cognition, yet this approach could obscure some domain-specific cognitive effects of statins. However, meta-analyses per cognitive domain were not suggestive of differences by statin treatment, and we are not aware of a postulated pathophysiological mechanism predicting domain-specific statin effects. Fourth, poor reporting in RCTs may explain why we found no evidence for an effect of statins on adverse events. ${ }^{75}$ However, we drew our main conclusion on the basis of pre-specified, sensitive measurements of cognition. Another possible limitation is that the results of the meta-analysis (although unlikely to differ greatly) apply to the statins for which there were trials with cognitive test outcomes-which would exclude fluvastatin, rosuvastatin, and pitavastatin.

\section{CONCLUSION}

Given these results, it is questionable whether the FDA class warning about potential cognitive adverse effects of statins is still warranted. Future post marketing surveillance efforts should focus on critical analysis of effects of re-challenge, as well as factors not addressed in statin clinical trials, such as excessively high dosage and compliance with guidelines. At the present time, our findings, combined with individual information about dosing and compliance, will allow physicians to more effectively counsel patients about their cognitive health concerns. Our findings are congruent with the recent 2013 American College of Cardiology/American Heart Association Cholesterol Guideline safety statement that, "for individuals presenting with a confusional state or memory impairment while on statin therapy, it may be reasonable to evaluate the patient for non-statin causes, such as exposure to other drugs, as well as for systemic and neuropsychiatric causes, in addition to the possibility of adverse effects associated with statin drug therapy."76

Acknowledgements: This study was partially supported by grant \#K08 HSO17735 to Dr. Daiello from the Agency for Healthcare Research and Quality (AHRQ). The results of this study were partially reported at the Alzheimer's Association International Conference in Boston on 16 July 2013.

Conflicts of interest: During the past 36 months, the authors have had no conflicts of interest relevant to this work. Dr. Ott received grant support for clinical trials in Alzheimer's disease from Lilly, Avid, Roche, Baxter, TauRx, Merck, Pfizer and Jannsen; grant support for cognitive instrument development from Univita; and consultant support as Data Safety Monitoring Board member for Accera.

Corresponding Author: Brian R. Ott, MD; Alzheimer's Disease Memory Disorders Center Rhode Island Hospital, APC 7, 593 Eddy Street, Providence, RI 02903, USA (e-mail: BOtt@brown.edu).

\section{REFERENCES}

1. FDA. FDA Drug Safety Communication: Important safety label changes to cholesterol-lowering statin drugs. 2-28-2012. 11-28-2012. Ref Type: Internet Communication

2. Benito-Leon J, Louis ED, Vega S, Bermejo-Pareja F. Statins and cognitive functioning in the elderly: a population-based study. J Alzheimers Dis. 2010;21(1):95-102.

3. Bettermann K, Arnold AM, Williamson J, et al. Statins, risk of dementia, and cognitive function: secondary analysis of the ginkgo evaluation of memory study. J Stroke Cerebrovasc Dis. 2012;21(6):436-44.

4. Beydoun MA, Beason-Held LL, Kitner-Triolo MH, et al. Statins and serum cholesterol's associations with incident dementia and mild cognitive impairment. J Epidemiol Community Health. 2011;65(11):949-57.

5. Evans MA, Golomb BA. Statin-associated adverse cognitive effects: survey results from 171 patients. Pharmacotherapy. 2009;29(7):800-11.

6. Orsi A, Sherman $\mathbf{O}$, Woldeselassie $\boldsymbol{Z}$. Simvastatin-associated memory loss. Pharmacotherapy. 2001;21(6):767-9.

7. Parker BA, Polk DM, Rabdya V, et al. Changes in memory function and neuronal activation associated with atorvastatin therapy. Pharmacotherapy. 2010;30(6):236e-40e.

8. Wagstaff LR, Mitton MW, Arvik BM, Doraiswamy PM. Statin-associated memory loss: analysis of 60 case reports and review of the literature. Pharmacotherapy. 2003;23(7):871-80. 
9. Zamrini E, McGwin G, Roseman JM. Association between statin use and Alzheimer's disease. Neuroepidemiology. 2004;23(1-2):94-8.

10. Zandi PP, Sparks DL, Khachaturian AS, et al. Do statins reduce risk of incident dementia and Alzheimer disease? The Cache County Study. Arch Gen Psychiatry. 2005;62(2):217-24.

11. Zhou B, Teramukai S, Fukushima M. Prevention and treatment of dementia or Alzheimer's disease by statins: a meta-analysis. Dement Geriatr Cogn Disord. 2007;23(3):194-201.

12. Feldman HH, Doody RS, Kivipelto M, et al. Randomized controlled trial of atorvastatin in mild to moderate Alzheimer disease: LEADe. Neurology. 2010;74(12):956-64

13. Muldoon MF, Barger SD, Ryan CM, et al. Effects of lovastatin on cognitive function and psychological well-being. Am J Med. 2000;108(7):538-46.

14. Muldoon MF, Ryan CM, Sereika SM, Flory JD, Manuck SB. Randomized trial of the effects of simvastatin on cognitive functioning in hypercholesterolemic adults. Am J Med. 2004;117(11):823-9.

15. Trompet S, van Vliet $\mathbf{P}$, de Craen $\mathbf{A J}$, et al. Pravastatin and cognitive function in the elderly. Results of the PROSPER study. J Neurol. 2010;257(1):85-90.

16. Padala KP, Padala PR, McNeilly DP, Geske JA, Sullivan DH, Potter JF. The effect of HMG-CoA reductase inhibitors on cognition in patients with Alzheimer's dementia: a prospective withdrawal and rechallenge pilot study. Am J Geriatr Pharmacother. 2012;10(5):296-302.

17. Rojas-Fernandez CH, Cameron JC. Is statin-associated cognitive impair ment clinically relevant? A narrative review and clinical recommendations. Ann Pharmacother. 2012;46(4):549-57.

18. Jukema JW, Cannon CP, de Craen AJ, Westendorp RG, Trompet S. The controversies of statin therapy: weighing the evidence. J Am Coll Cardiol. 2012;60(10):875-81

19. Macedo AF, Taylor FC, Casas JP, Adler A, Prieto-Merino D, Ebrahim S. Unintended effects of statins from observational studies in the general population: systematic review and meta-analysis. BMC Med. 2014;12:51.

20. Wong WB, Lin Vw, Boudreau D, Devine EB. Statins in the prevention of dementia and Alzheimer's disease: a meta-analysis of observational studies and an assessment of confounding. Pharmacoepidemiol Drug Saf. 2013;22(4):345-58

21. Swiger KJ, Manalac RJ, Blumenthal RS, Blaha MJ, Martin SS. Statins and cognition: a systematic review and meta-analysis of short- and longterm cognitive effects. Mayo Clin Proc. 2013;88(11):1213-21.

22. Richardson $\mathbf{K}$, Schoen $\mathbf{M}$, French B, et al. Statins and cognitive function: a systematic review. Ann Intern Med. 2013;159(10):688-97.

23. McGuinness B, Craig D, Bullock R, Passmore P. Statins for the prevention of dementia. Cochrane Database Syst Rev. 2009;2, CD003160.

24. McGuinness B, O'Hare J, Craig D, Bullock R, Malouf R, Passmore P. Statins for the treatment of dementia. Cochrane Database Syst Rev. 2010;8:CD007514

25. Taylor F, Ward $\mathbf{K}$, Moore TH, et al. Statins for the primary prevention of cardiovascular disease. Cochrane Database Syst Rev. 2011;1:CD004816.

26. Naci H, Brugts J, Ades T. Comparative tolerability and harms of individual statins: a study-level network meta-analysis of 246955 participants from 135 randomized, controlled trials. Circ Cardiovasc Qual Outcome. 2013;6(4):390-9

27. Wallace BC, Small K, Brodley CE, Lau J, Trikalinos TA. Deploying an interactive machine learning system in an evidence-based practice center: abstrackr. 2012; p. 819-24.

28. da Costa BR, Nuesch E, Rutjes AW, et al. Combining follow-up and change data is valid in meta-analyses of continuous outcomes: a metaepidemiological study. J Clin Epidemiol. 2013;66(8):847-55.

29. Cochrane handbook for systematic reviews of interventions. In: Higgins JPT, ed. Cochrane Collaboration. version 5.0.0 ed. 2008.

30. Mohs RC. The Alzheimer's Disease Assessment Scale. Int Psychogeriatr. 1996;8(2): 195-203.

31. Folstein MF, Folstein SE, McHugh PR. Mini-mental state. A practical method for grading the cognitive state of patients for the clinician. J Psychiatr Res. 1975; 12(3): 189-98.

32. Hedges LV. Distribution theory for Glass's estimator of effect size and related estimators. J Educ Stat. 1981;6(2):107-28.

33. Van Den Noortgate W, Onghena P. Estimating the mean effect size in meta-analysis: bias, precision, and mean squared error of different weighting methods. Behav Res Methods Instrum Comput. 2003;35(4):504-11

34. Hedges LV, Tipton E, Johnson MC. Robust variance estimation in metaregression with dependent effect size estimates. Res Synth Meth 2010;1(1):39-65.
35. Sierra S, Ramos MC, Molina P, Esteo C, Vazquez JA, Burgos JS. Statins as neuroprotectants: a comparative in vitro study of lipophilicity, bloodbrain-barrier penetration, lowering of brain cholesterol, and decrease of neuron cell death. J Alzheimers Dis. 2011;23(2):307-18.

36. MRC/BHF Heart Protection Study of cholesterol lowering with simvastatin in 20,536 high-risk individuals: a randomised placebo-controlled trial. Lancet 2002 July 6;360(9326):7-22.

37. Berk-Planken I, de Konig I, Stolk R, Jansen H, Hoogerbrugge $\mathbf{N}$. Atorvastatin, diabetic dyslipidemia, and cognitive functioning. Diabetes Care. 2002;25(7):1250-1.

38. Carlsson CM, Papcke-Benson $\mathrm{K}$, Carnes M, McBride PE, Stein JH. Health-related quality of life and long-term therapy with pravastatin and tocopherol (vitamin E) in older adults. Drugs Aging. 2002;19(10):793805.

39. Carlsson CM, Gleason CE, Hess TM, et al. Effects of simvastatin on cerebrospinal fluid biomarkers and cognition in middle-aged adults at risk for Alzheimer's disease. J Alzheimers Dis. 2008;13(2):187-97.

40. Collins R, Armitage J, Parish S, Sleight P, Peto R. Effects of cholesterollowering with simvastatin on stroke and other major vascular events in 20536 people with cerebrovascular disease or other high-risk conditions. Lancet. 2004;363(9411):757-67.

41. Cutler N, Sramek J, Veroff A, Block G, Stauffer L, Lines C. Effects of treatment with simvastatin and pravastatin on cognitive function in patients with hypercholesterolaemia. Br J Clin Pharmacol. 1995;39(3):333-6.

42. FDA. CRESTOR® (rosuvastatin calcium) NDA 21-366/S-016. Briefing Document for Endocrinologic and Metabolic Drugs Advisory Committee Meeting of December 15, 2009. FDA website 2013;Available at: URL: http://www.fda.gov/downloads/AdvisoryCommittees/CommitteesMeetingMaterials/Drugs/EndocrinologicandMetabolicDrugsAdvisoryCommittee/UCM193833.pdf.

43. Gengo F, Cwudzinski D, Kinkel P, Block G, Stauffer L, Lines C. Effects of treatment with lovastatin and pravastatin on daytime cognitive performance. Clin Cardiol. 1995;18(4):209-14

44. Gibellato MG, Moore JL, Selby $\mathbf{K}$, Bower EA. Effects of lovastatin and pravastatin on cognitive function in military aircrew. Aviat Space Environ Med. 2001;72(9):805-12.

45. Harrison RW, Ashton CH. Do cholesterol-lowering agents affect brain activity? A comparison of simvastatin, pravastatin, and placebo in healthy volunteers. Br J Clin Pharmacol. 1994;37(3):231-6.

46. Hsia J, MacFadyen JG, Monyak J, Ridker PM. Cardiovascular event reduction and adverse events among subjects attaining low-density lipoprotein cholesterol $<50 \mathrm{mg} / \mathrm{dl}$ with rosuvastatin. The JUPITER trial (Justification for the Use of Statins in Prevention: an Intervention Trial Evaluating Rosuvastatin). J Am Coll Cardiol. 2011;57(16):1666-75.

47. JUPITER, Roberts MD. Endocrinologic and Metabolic Drug Advisory Committee Meeting. http://www.fdagov/downloads/advisorycommittees / committeesmeetingmaterials/drugs / endocrinologicandmetabolicdrugsadvisorycommittee/ucm194918pdf 2012. AccessedNovember 2, 2014.

48. Kostis JB, Rosen RC, Wilson AC. Central nervous system effects of HMG CoA reductase inhibitors: lovastatin and pravastatin on sleep and cognitive performance in patients with hypercholesterolemia. J Clin Pharmacol. 1994;34(10):989-96.

49. Krab LC, de Goede-Bolder A, Aarsen FK, et al. Effect of simvastatin on cognitive functioning in children with neurofibromatosis type 1: a randomized controlled trial. JAMA. 2008;300(3):287-94.

50. Posvar EL, Radulovic LL, Cilla DD Jr, Whitfield LR, Sedman AJ. Tolerance and pharmacokinetics of single-dose atorvastatin, a potent inhibitor of HMG-CoA reductase, in healthy subjects. J Clin Pharmacol. 1996;36(8):728-31.

51. Ridker PM, Danielson E, Fonseca FA, et al. Rosuvastatin to prevent vascular events in men and women with elevated C-reactive protein. N Engl J Med. 2008;359(21):2195-207.

52. Roth T, Richardson GR, Sullivan JP, Lee RM, Merlotti L, Roehrs T. Comparative effects of pravastatin and lovastatin on nighttime sleep and daytime performance. Clin Cardiol. 1992;15(6):426-32.

53. Sanchez-Aguilar M, Tapia-Perez JH, Sanchez-Rodriguez JJ, et al. Effect of rosuvastatin on cytokines after traumatic head injury. J Neurosurg. 2013;118(3):669-75.

54. Sano M, Bell KL, Galasko D, et al. A randomized, double-blind, placebocontrolled trial of simvastatin to treat Alzheimer disease. Neurology. 2011;77(6):556-63.

55. Santanello NC, Barber BL, Applegate WB, et al. Effect of pharmacologic lipid lowering on health-related quality of life in older persons: results from 
the cholesterol reduction in seniors program (CRISP) pilot study. J Am Geriatr Soc. 1997;45(1):8-14.

56. Shepherd J, Blauw GJ, Murphy MB, et al. Pravastatin in elderly individuals at risk of vascular disease (PROSPER): a randomised controlled trial. Lancet. 2002;360(9346): 1623-30.

57. Simons M, Schwarzler F, Lutjohann D, et al. Treatment with simvastatin in normocholesterolemic patients with Alzheimer's disease: A 26-week randomized, placebo-controlled, double-blind trial. Ann Neurol. 2002;52(3):346-50.

58. Sparks DL, Sabbagh MN, Connor DJ, et al. Atorvastatin for the treatment of mild to moderate Alzheimer disease: preliminary results. Arch Neurol. 2005;62(5):753-7.

59. Sparks DL, Connor DJ, Sabbagh MN, Petersen RB, Lopez J, Browne P. Circulating cholesterol levels, apolipoprotein E genotype and dementia severity influence the benefit of atorvastatin treatment in Alzheimer's disease: results of the Alzheimer's Disease Cholesterol-Lowering Treatment (ADCLT) trial. Acta Neurol Scand Suppl. 2006; 185:3-7.

60. Sparks DL, Sabbagh M, Connor D, et al. Statin therapy in Alzheimer's disease. Acta Neurol Scand Suppl. 2006;185:78-86.

61. Sparks DL, Lemieux SK, Haut MW, et al. Hippocampal volume change in the Alzheimer disease cholesterol-lowering treatment trial. Cleve Clin J Med. 2008;75(Suppl 2):S87-S93.

62. Summers MJ, Oliver KR, Coombes JS, Fassett RG. Effect of atorvastatin on cognitive function in patients from the lipid lowering and onset of renal disease (LORD) trial. Pharmacotherapy. 2007;27(2):183-90.

63. Tapia-Perez J, Sanchez-Aguilar M, Torres-Corzo JG, et al. Effect of rosuvastatin on amnesia and disorientation after traumatic brain injury (NCTO03229758). J Neurotrauma. 2008;25(8):1011-7.

64. Tendolkar I, Enajat M, Zwiers MP, et al. One-year cholesterol lowering treatment reduces medial temporal lobe atrophy and memory decline in stroke-free elderly with atrial fibrillation: evidence from a parallel group randomized trial. Int J Geriatr Psychiatry. 2012;27(1):49-58.

65. Schrag A, Schott JM. What is the clinically relevant change on the ADASCog? J Neurol Neurosurg Psychiatry. 2012;83(2):171-3.
66. Simpson RJ Jr, Mendys P. The effects of adherence and persistence on clinical outcomes in patients treated with statins: a systematic review. J Clin Lipidol. 2010;4(6):462-71.

67. Vural K, Tuglu MI. Neurotoxic effect of statins on mouse neuroblastoma NB2a cell line. Eur Rev Med Pharmacol Sci. 2011;15(9):985-91.

68. MacDonald JS, Gerson RJ, Kornbrust DJ, et al. Preclinical evaluation of lovastatin. Am J Cardiol. 1988;62(15):16J-27J.

69. Stuart SA, Robertson JD, Marrion NV, Robinson ES. Chronic pravastatin but not atorvastatin treatment impairs cognitive function in two rodent models of learning and memory. PLoS One. 2013;8(9):e75467.

70. Elkind MS, Sacco RL, Macarthur RB, et al. High-dose lovastatin for acute ischemic stroke: results of the phase I dose escalation neuroprotection with statin therapy for acute recovery trial (NeuSTART). Cerebrovasc Dis. 2009;28(3):266-75.

71. Cuciureanu M, Vlase L, Muntean D, Varlan I, Cuciureanu R. Grapefruit juice-drug interactions: importance for pharmacotherapy. Rev Med Chir Soc Med Nat Iasi. 2010;114(3):885-91.

72. Hare JT, Elliott DP. Grapefruit juice and potential drug interactions. Consult Pharm. 2003;18(5):466-72.

73. Goh IX, How CH, Tavintharan S. Cytochrome P450 drug interactions with statin therapy. Singap Med J. 2013;54(3):131-5.

74. Leung A, Schaefer EW, Tempelhof MW, Stone NJ. Emphasizing statin safety in the hospitalized patient: a review. Am J Med. 2012;125(9):845-53.

75. Ioannidis JP, Lau J. Completeness of safety reporting in randomized trials: an evaluation of 7 medical areas. JAMA. 2001;285(4):43743.

76. Stone NJ, Robinson J, Lichtenstein AH et al. 2013 ACC/AHA Guideline on the Treatment of Blood Cholesterol to Reduce Atherosclerotic Cardiovascular Risk in Adults: A Report of the American College of Cardiology/American Heart Association Task Force on Practice Guidelines. Circulation 2013 November 12. 